\title{
Pulmonary artery tumour-embolism diagnosed by endobronchial ultrasound-guided transbronchial needle aspiration
}

\section{To the Editors:}

A 61-yr-old female was diagnosed with pulmonary embolism. She had a history of poorly differentiated thyroid cancer with follicular cell phenotype, and had first been treated in 2006 by thyroidectomy and radioactive iodine-131. Local relapse (cervical nodes) had occurred within 3 months and required five cycles of chemotherapy, consisting of cisplatin and doxorubicin, followed by cervical radiotherapy and surgical resection of residual nodes. 2 yrs later during follow-up, abnormal ${ }^{18} \mathrm{~F}$ fluorodeoxyglucose (FDG) uptake was described on positron emission tomography (PET)/computed tomography (CT) in the left thoracic hilar regions. CT pulmonary angiography showed a filling defect in the left lower lobe pulmonary artery and at the origin of the lingular artery; no delayed embolism enhancement was observed. It was considered to be a pulmonary embolism, and anticoagulation was initiated. 6 months later, follow-up imaging showed stability of both FDG uptake and CT pulmonary angiography images, and anticoagulation was continued. 10 months later, follow-up showed a right pulmonary upper lobe nodule on CT scan associated with high FDG uptake on $\mathrm{PET} / \mathrm{CT}$, and thyroglobulin ascension. This nodule was considered to be a thyroid cancer metastasis and treated by CyberKnife radiotherapy. As FDG uptake and thyroglobulin levels remained high despite radiotherapy, a complementary treatment by radio frequency ablation was administered, leading to the disappearance of the right pulmonary upper lobe nodule. Thyroglobulin levels remained high nonetheless. A subsequent CT scan showed a persistent filling defect in the left lower lobe pulmonary artery and at the origin of the lingular artery, despite over 12 months of anticoagulation (fig. 1). No delayed thrombus enhancement was seen on this occasion either. At this time, a)

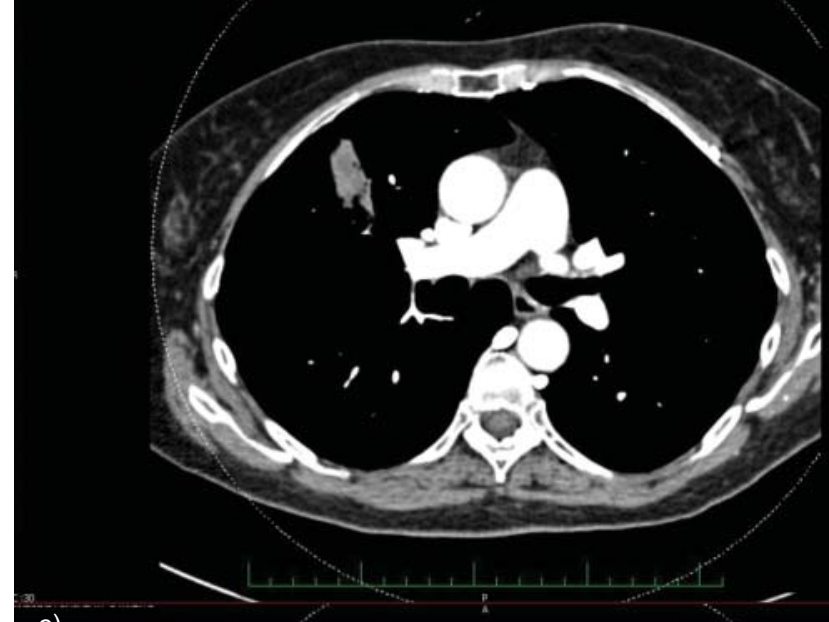

c) b)

d)

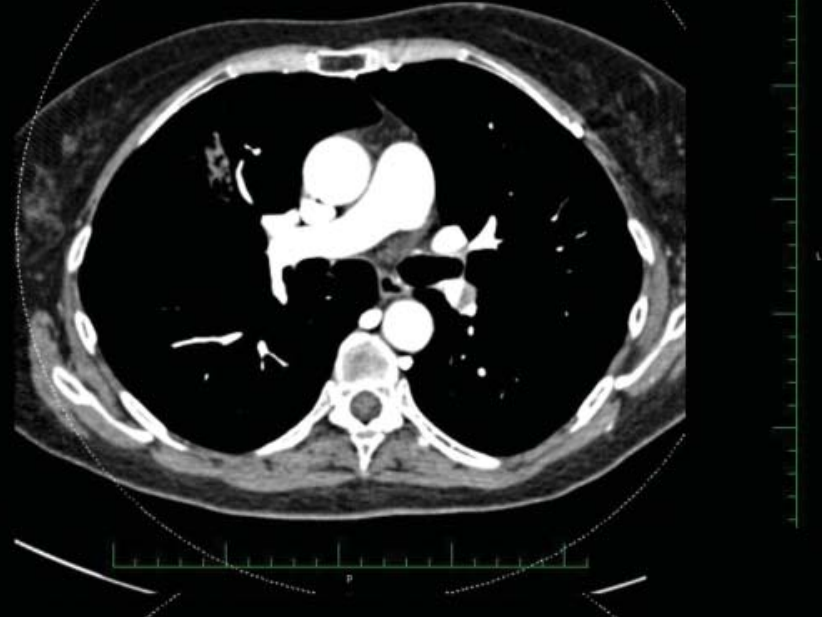

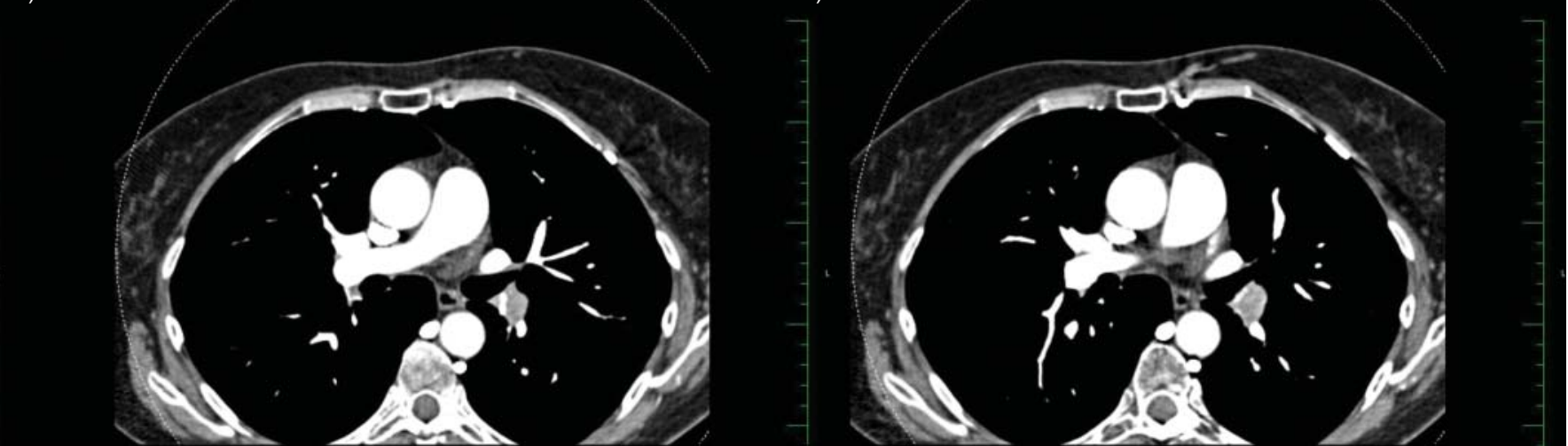

FIGURE 1. Computed tomography pulmonary angiography showing persistent filling defect in the left lower lobe pulmonary artery, 12 months after treatment (a-d). 
metastatic embolism of the left lower lobe pulmonary artery was strongly suspected, but had not been confirmed. To obtain histological diagnosis, an endobronchial ultrasound-guided transbronchial needle aspiration (EBUS-TBNA) was selected as the diagnostic technique of choice. EBUS-TBNA was performed under general anaesthesia and showed an exclusively endovascular hyperechoic image in the left lower lobe pulmonary artery, suggesting an abnormal lesion (fig. 2), which was aspirated. Histological findings revealed follicular thyroid cancer cells with cytoplasmic thyroglobulin antibody fixation. The patient was referred to a thoracic surgery centre and the case was reviewed by a multidisciplinary team; surgical exploration was decided upon. Thoracotomy was performed and macroscopic findings showed an exclusively endovascular metastatic invasion of the left lower lobe pulmonary artery and at the origin of the lingular artery, without any abnormal extravascular lesion. It was considered that pneumonectomy could not be avoided, so it was performed without any complications. Histopathology of the resected tissue confirmed macroscopic findings: exclusive metastatic embolism of follicular thyroid cancer in left lower lobe pulmonary artery and at the origin of the lingular artery.

To our knowledge, this is the first report of thyroid cancer metastatic pulmonary embolism diagnosed by EBUS-TBNA.
This observation confirms the high diagnostic accuracy of EBUS-TBNA.

Metastases in pulmonary arteries were first described in 1897 by SCHMIDT [1]. They consist of exclusive neoplastic thromboses of arterial lumens without an adjacent nodule or tumour. Four types are described: neoplasic embolism of main arteries (which is the case reported), carcinomatous lymphangitis, thrombotic microangiopathy, and association of carcinomatous lymphangitis and thrombotic microangiopathy [2]. The first descriptions of this entity were essentially from autopsy series. Incidence in a series of deceased patients was estimated to be from 10 to $26 \%$ [3]. The types of primary cancers often found to be responsible for metastatic embolism include (in descending order): hepatocellular carcinomas, choriocarcinomas, endocrine gland carcinomas (such as thyroid cancer), biliary tract cancers, renal cancers, sarcomas, breast cancers and pancreatic cancer $[4,5]$. However, all types of cancers may be involved. The diagnosis of metastatic pulmonary embolism is difficult to establish, and often only diagnosed at autopsy. Clinical features are nonspecific and are quite similar to those of thrombotic pulmonary embolism, e.g. dyspnoea is present in $50 \%$ of cases. Metastatic embolism must be suspected when no results are seen after extended anticoagulant therapy and regression is seen with the addition of chemotherapy. Differences between

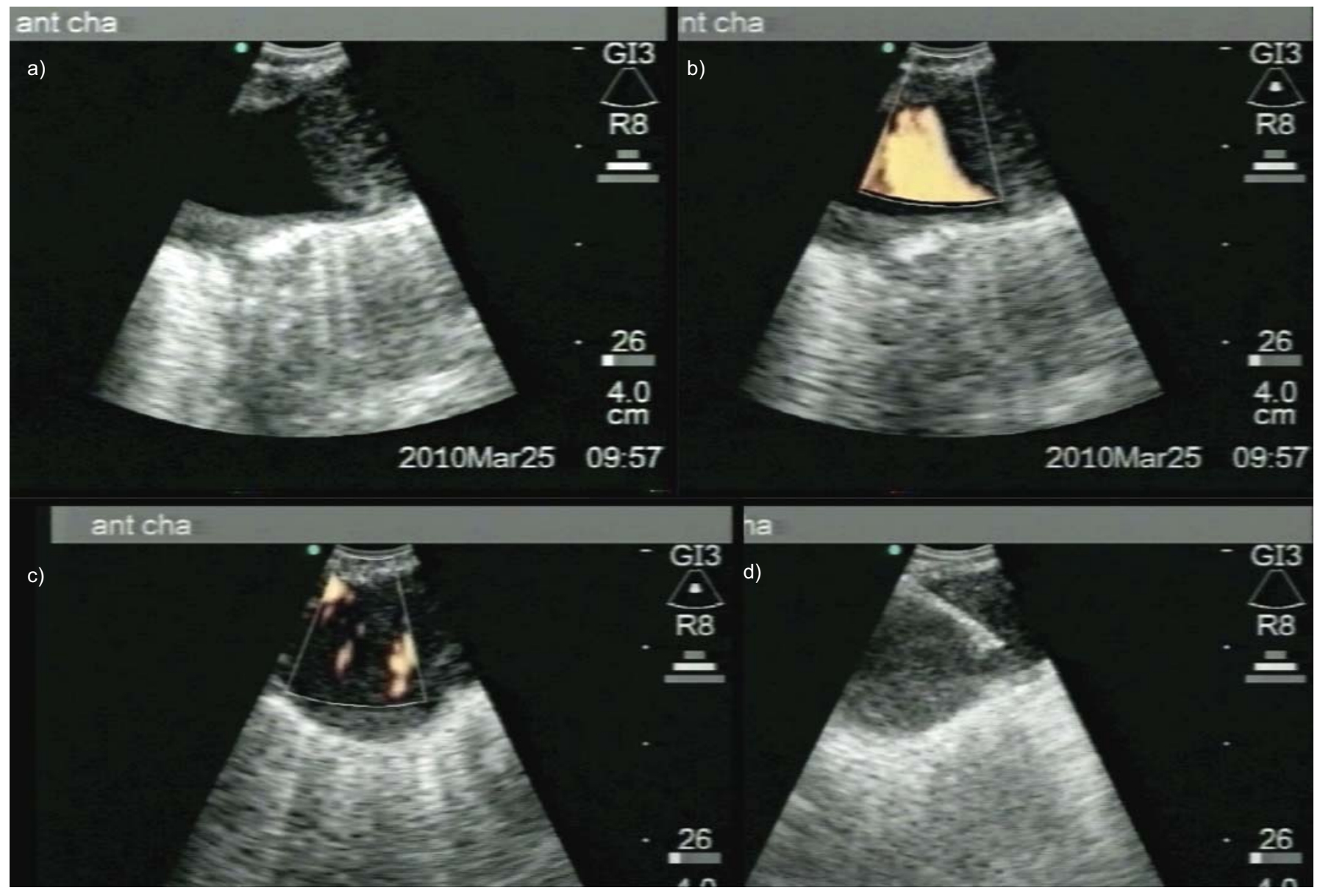

FIGURE 2. Exclusively endovascular hyperechoic image in left lower lobe pulmonary artery. 
thrombotic and metastatic pulmonary embolism are not easily identified on radiographic and CT scan findings, making radiological diagnosis difficult. In patients with prior cancer history, CT scan can show excessive enlargement of pulmonary arteries and thrombus enhancement on delayed phase scans, or embolism calcification, especially in chondrosarcoma or osteosarcoma [6]. In a retrospective series of four patients with pulmonary intravascular metastasis, a CT scan showed multifocal dilatation and beading of peripheral pulmonary arteries, primarily in a subsegmental distribution involving multiple lobes. The authors concluded that these images were highly suggestive of metastatic intravascular embolism [7]. Recently, a "tree-in-bud" appearance has been described on thin-section $\mathrm{CT}$, which is probably related to the presence of cancer cells in centrilobular arteries [8].

PET/CT FDG was positive in our case, but it has been shown that PET/CT is not sensitive enough to differentiate metastatic from thrombotic embolism [9].

Obtaining histological diagnosis is sometimes possible through transthoracic biopsy or endovascular biopsy using pulmonary artery catheterisation, but patients have to be referred to a specialised centre. In most cases, diagnosis is only suspected and unfortunately confirmed by autopsy. Although EBUS-TBNA was first developed for histological diagnosis in primary or secondary lung cancer, and for mediastinal lymph node staging, its increasing use leads to more and more new applications [10]. We recently reported a case of thrombotic pulmonary embolism diagnosed during an endobronchial ultrasound procedure [11].

EBUS-TBNA is a simple and safe procedure to obtain histological diagnosis of metastatic pulmonary embolism.

\section{A.L. Blanc*, C. Jardin*, J.B. Faivre", O. Le Rouzic*, C. Do Cao", L. Benhamed ${ }^{+}$, P.P. Ramon* and C. Fournier*}

*Service d'Endoscopie Respiratoire, Pôle des Maladies Respiratoires, "Service de Radiologie Thoracique, Pôle d'Imagerie, +Service de Chirurgie Thoracique, Hôpital Calmette, and "Service de Médecine Interne et Endocrinologie, Hopital Claude Huriez, CHRU Lille, Lille, France.
Correspondence: C. Fournier, Service d'Endoscopie Respiratoire, Bd du Pr Leclerc, CHU de Lille - Hopital Calmette, 59037 Lille cedex, France. E-mail: clement.fournier@chru-lille.fr

Statement of Interest: Statements of interest for P.P. Ramon and C. Fournier can be found at www.erj.ersjournals.com/site $/ \mathrm{misc} /$ statements.xhtml

\section{REFERENCES}

1 Schmidt W. Ueber Krebszellenembolien in den Lugenarterien. Zentrabl Allg Path 1897; 8: 868.

2 Roberts KE, Hamele-Bena D, Saqi A, et al. Pulmonary tumor embolism: a review of the literature. Am J Med 2003; 115: 228-232.

3 Winterbauer RH, Elfenbein IB, Ball WC. Incidence and clinical significance of tumor embolization to the lungs. Am J Med 1968; 45: 271-290.

4 Chomette G, Auriol M, Sevestre C, et al. Metastatic pulmonary emboli: clinical types and incidence. Ann Med Intern 1980; 13: 217-221.

5 Grass H, Schuff A, Staak M, et al. Tumor embolism as a cause of an unexpected death: a case report. Pathol Res Pract 2003; 199: 349-352.

6 Hauret L, Minvielle F, Ehre P, et al. Metastasis in pulmonary arteries. J Radiol 2000; 81: 807-809.

7 Shepard JA, Moore EH, Templeton PA, et al. Pulmonary intravascular tumor emboli: dilated and beaded peripheral pulmonary arteries at CT. Radiology 1993; 187: 797-801.

8 Han D, Lee KS, Franquet T, et al. Thrombotic and nonthrombotic pulmonary arterial embolism: spectrum of imaging findings. Radiographics 2003; 23: 1521-1539.

9 Goethals I, Smeets P, De Winter O, et al. Focally enhanced F-18 fluorodeoxyglucose (FDG) uptake in incidentally detected pulmonary embolism on PET/CT scanning. Clin Nucl Med 2006; 31: 497-498.

10 Aumiller J, Herth FJF, Krasnik M, et al. Endobronchial ultrasound for detecting central pulmonary emboli: a pilot study. Respiration 2009; 77: 298-302.

11 Le Rouzic O, Tercé G, Jardin C, et al. Pulmonary embolism diagnosed during an endobronchial ultrasound procedure. Rev Mal Respir 2010; 27: 775-777. 